\title{
Cuidado y responsabilidad
}

NATACHA BORGEAUD-GARCIANDÍA ${ }^{I}$

\section{Introducción}

$\int$

N UNA INVESTIGACIÓN sobre el trabajo desarrollado por cuidadoras migrantes que trabajan sin retiro con personas mayores dependientes, el análisis de los afectos y las relaciones interpersonales entre los actores más íntimamente involucrados me llevó a desarrollar una reflexión sobre el papel de las "coacciones del corazón" (Morice, 2000) asociadas a lo que llamé "coacciones por responsabilidad" (hacia otros) que se experimentan simultáneamente como tantas "obligaciones morales" (para sí) (Borgeaud-Garciandía, 2017).

Profundizando la reflexión, se analiza cómo es vivida, interpretada, o inclusive utilizada la responsabilidad del cuidado que recae sobre estas trabajadoras, que ocupan posiciones sociales y empleos subalternos y por muchos aspectos extremos. Lejos de ser fácilmente descrita, delimitada y distribuida, la responsabilidad es objeto, tanto en el seno de la organización social del cuidado como de la relación entre instituciones y familias y dentro de las familias, de tensiones y acciones (delegación, asunción, omisión, ignorancia, etc.), vinculadas a posiciones diferenciadas de poder. Su distribución desigual y consiguiente responsabilización diferenciada (Biroli, 2015) se acompañan de una responsabilidad "vivida" (y padecida) intrínseca al cuidado como trabajo sobre lo humano, más en contextos de mayor dependencia y fragilidad. Sobre este aspecto de la responsabilidad deseamos profundizar.

Se ofrece, en una primera instancia, una descripción de la situación de cuidado analizada, realizado por cuidadoras migrantes en la ciudad de Buenos Aires (I). Después de eso, se propone una incursión de índole más teórica para situar mejor la perspectiva privilegiada por nuestro enfoque (II). Luego, se analizan sucesivamente: las relaciones entre responsabilidad y división del trabajo - a nivel social y luego micro sociológico - (III); entre responsabilidad y trabajo de cuidado (IV), y finalmente entre cuidado, responsabilidad, poder y dominación $(\mathrm{V})$. Esta interpretación de las relaciones entre cuidado y responsabilidad se apoya en los hallazgos empíricos y teóricos provenientes de la investigación realizada anteriormente mencionada.

\section{Vivir y trabajar con personas mayores muy dependientes}

La investigación se realizó en la capital argentina. Se basó en relatos biográficos de trabajadoras migrantes que cuidaban a personas mayores a tiempo completo o sin retiro, con el propósito de indagar en la complejidad del trabajo de cuidado y sus múltiples dimensiones, partiendo de la relación subjetiva que estas mujeres mantuvieron con sus experiencias. 
Los relatos reunidos provienen de mujeres, en su mayoría migrantes peruanas, pero también de Paraguay y Argentina, que llegaron a Buenos Aires en busca de trabajo para mejorar sus condiciones de vida y las de sus familias. En el caso de las mujeres migrantes peruanas entrevistadas, llegaron en la década de 1990, solas, en un contexto de crisis económica y política en su país. En Argentina, tanto por la desigual estructuración del mercado laboral como (una cosa está ligada a la otra) por el funcionamiento de las redes de migrantes, se insertan en los empleos domésticos y de cuidados. Las migraciones de mujeres argentinas y paraguayas entrevistadas se inscriben con mayor frecuencia en historias migratorias familiares y comunitarias y abarcan períodos de tiempo extendidos, marcados por numerosas idas y vueltas. En todos los casos, las cuidadoras habían pasado más de 10 años en la capital.

En el transcurso de sus trayectorias de trabajo en Buenos Aires, unas y otras habían trabajado (o siguen trabajando) "sin retiro" con adultos mayores dependientes. Si bien esta modalidad de empleo es más común durante las primeras etapas de la migración cuando las trabajadoras están solas, algunas de las cuidadoras "sin retiro" contaban con familia y vivienda. Inclusive, en algunos casos, se han especializado en este tipo de empleo, encontrando en él una forma de estabilización de su trayectoria.

Los trabajos de cuidado sin retiro con personas mayores altamente dependientes presentan una cierta diversidad, dependiendo de la persona o las personas asistidas, las patologías que sufren, el tipo de acuerdo sobre la organización del empleo, la presencia o (casi) ausencia de la familia, el desarrollo de cada situación. Sin embargo, un caso prototípico reúne a una trabajadora migrante con una mujer mayor que vive sola, y padece un proceso de deterioro físico, pero más aún mental (demencia senil o tipo Alzheimer), que empeora con el tiempo llevando a la cuidadora no poder dejar el espacio doméstico de la persona asistida durante días, hasta su receso semanal.

Desde el punto de vista del empleo, siguiendo las características del sector en Argentina, la mayoría de las cuidadoras no habían sido registradas. Esta situación ha cambiado un poco desde entonces, gracias a una política más incisiva de blanqueo del empleo doméstico por parte del gobierno. ${ }^{1}$ En relación con la situación migratoria, aunque la ilegalidad migratoria haya servido para justificar el no registro laboral, la posibilidad de regularizar su residencia ${ }^{2}$ parece tener menos peso en el respecto de sus derechos laborales que las condiciones generales que caracterizan al sector (marcado por su alta informalidad, precariedad y devaluación social).

Las cuidadoras trabajan jornadas completas o sin retiro, teóricamente desde el domingo por la noche o el lunes por la mañana hasta el sábado por la tarde. La organización del empleo se relaciona con las necesidades de la persona asistida. Esta última, dependiente y muchas veces afectivamente ligada a la cuidadora y sus cuidados, vive con dificultad los cambios y sus ausencias, inclusive 
semanales. Los empleos suelen terminarse con la muerte o institucionalización de la persona asistida. El trabajo de cuidado no se encuentra pautado tanto por la organización del empleo, como por el ritmo de la convivencia, las necesidades y la dependencia.

Las condiciones de trabajo son particularmente difíciles, hasta el punto en que las calificamos como extremas, reflejando de manera exacerbada las dimensiones y los efectos del trabajo de cuidado sobre las cuidadoras. Generalmente, las familias recurren a una cuidadora sin retiro cuando sus padres ya no pueden vivir solos, para evitar o retrasar su institucionalización. Cuanto más aumenta la dependencia, menor es la posibilidad de ausentarse, llegando a situaciones de encierro con sus efectos nocivos (Borgeaud-Garciandía, 2013). El trabajo de cuidado se inscribe en la intimidad de los hogares y de los cuerpos. La intrusión en el hogar que "equivale a una intrusión en la intimidad corporal", en contacto con cuerpos disfóricos fuentes de espanto y rechazo (Memmi, 2016). Es un cuerpo que deja de ser unidad y frontera, evidenciando su vulnerabilidad, fuente de emociones (asco, miedo, angustia) que la cuidadora debe poder controlar y redefinir rápidamente (Borgeaud-Garciandía, 2016, 2017). Las cuidadoras trabajan a la sombra de la muerte, y acompañan a la enfermedad. Cuando las personas mayores sufren de demencia senil, se alteran las referencias compartidas, ante lo cual la cuidadora busca reconocer el universo de la persona asistida, acompañarla lo mejor posible, protegiéndose del agotamiento físico y emocional. Cuando se combinan el encierro, el agotamiento, la dependencia profunda y la demencia, el trabajo de cuidado lleva a las trabajadoras a los límites de su propia capacidad de resistencia.

\section{Cuidado y responsabilidad contextualizada}

A lo largo de la investigación, se buscó analizar este trabajo de gran complejidad, las maneras de realizarlo, de relacionarse con él, de darle sentido, de protegerse de él, el papel de los afectos y el peso del sentimiento de responsabilidad que reenvía -incluso cuando éste se encuentra impuesto y constreñido por relaciones de poder desiguales- a la construcción de una ética de la responsabilidad.

Cuando Carol Gilligan (1982) desarrolla los primeros análisis que darán paso a las teorías del cuidado, destaca el rol de la responsabilidad, no desde la óptica liberal de la justica, sino como un elemento que desarrollan los actores morales insertos en relaciones concretas de interdependencia y que constituye una dimensión central de la ética del cuidado. Lejos de referirse a un concepto abstracto, la responsabilidad integra los vínculos que tenemos con los demás, en tanto no somos entidades aisladas sino sujetos relacionales, colocados en el centro de un conjunto complejo de relaciones. Estas relaciones implican obligaciones morales que se asumen, ignoran, delegan, olvidan, priorizan, descuidan etc., y que pueden entrar en tensión (como aquella cuidadora que cuida una adulta mayor mientras sus hijos quedan solos al cuidado unos de otros, o aquella otra 
que termina llevando a su pequeño hijo a vivir con ella y la anciana que cuida, a escondidas de la hija de esta última). En este sentido, tal como recuerda Tronto (2013), las relaciones son particulares y el análisis debe ser contextualizado.

Este enfoque relacional abre varias pistas para la reflexión:

Una primera reflexión tiene que ver con el sujeto que se considera. El sujeto en relación se aleja del ideal de sujeto soberano y autónomo, motivado por el interés individual, "homo œconomicus" cuyas relaciones serían reguladas por el mercado, para reconocer un sujeto inmediatamente vinculado a los demás, y por ello mismo, dependiente y vulnerable. Pulcini nos recuerda que los temas de fragilidad y vulnerabilidad del sujeto representan temas contemporáneos de reflexión, desde diferentes enfoques que tienen en común "la idea que la parábola del individualismo moderno y del sujeto soberano terminó ocultando, o, mejor dicho, desembocó en una represión de esta condición ontológica de vulnerabilidad que, una vez admitida, puede llevar al sujeto a reconocer su insuficiencia y dependencia con los demás, el hecho de que se encuentra inevitablemente vinculado a otros, a otras vidas, a otros destinos" (Pulcini, 2012, p.55). Reconocer en la vulnerabilidad de otro la de uno mismo desempeñaría un papel fundamental en las relaciones de cuidado. Por un lado, permite la apertura hacia el otro, necesaria para poder ir hacia él. Por otro, y a diferencia de un esquema dicotómico y unilateralista que opone un dador de cuidados activo y un receptor de cuidados, dependiente y pasivo, tal enfoque introduce una forma de reciprocidad a pesar de la asimetría. Siguiendo a Gros (2007), la solicitud (pensada en la relación de cuidado curativo) abre el sujeto a la posibilidad de recibir algo del otro. Así, la "construcción ética de la solicitud" se refiere al concepto de responsabilidad ética o, siguiendo a Levinas, de "responsabilidad para con el otro" en respuesta a la confrontación con fragilidad de ese otro (ibidem, p.20). La idea de construcción es fundamental para nuestro enfoque. Porque entre estos enfoques teóricos y la confrontación real con el cuidado existe un trabajo, real, concreto, necesario hecho sobre uno mismo y sobre la relación de cuidado para lograr responder (retomando aquí el origen etimológico de la palabra responsabilidad) a las necesidades de la dependencia. La solicitud y la responsabilidad sentidas, así como los significados que tienen, no son dadas y naturales, sino que se trabajan cotidianamente y permanecen frágiles.

Otra pista de reflexión planteada por Tronto, se refiere a las diferentes capacidades que tienen los actores para actuar frente a la responsabilidad, en situaciones en las cuales las relaciones que los unen son a menudo asimétricas. Las relaciones, los cuidados, las responsabilidades se encuentran atravesados por relaciones de poder. "Cuando empezamos a percibir que nuestras actitudes ante la responsabilidad - el hecho de asignar una responsabilidad, de aceptarla, de postergarla, de evitarla o de cumplir con ella - están asociadas a posiciones de poder, entendemos que los agentes no son iguales ante la responsabilidad" (Tronto, 2013, p.112). No abarca únicamente relaciones directas, sino redes de relaciones que involu- 
cran a individuos, instituciones y Estados en relaciones complejas y conflictivas. En otras palabras, aunque las relaciones personales de cuidado pueden ser objeto de estudios micro sociológicos, no se desvinculan de la organización social de los cuidados en la que están inmersos y dependen. Más cerca de las experiencias vividas, sin embargo, es posible alejarse de un patrón dominante / dominado para capturar los juegos y problemáticas que estructuran las relaciones concretas.

Un tercer tema, presente en los anteriores, se refiere a la necesidad de partir del terreno áspero de la experiencia ordinaria de trabajo de cuidado para poder captar qué significa la responsabilidad del cuidado, las dificultades que plantea, las formas que reviste, las resistencias y estrategias que genera, para no caer en una ética normativa e ideal - e inclusive idealista - de la responsabilidad, que desconocería la mirada de los protagonistas del cuidado, la rudeza y efectos dañinos de ese trabajo, su aspecto defensivo, y que, por otra parte, menospreciaría la dimensión colectiva y política del trabajo que no puede, tal como lo recuerda Garrau (2009), descansar únicamente en las actitudes del cuidador.

\section{Cuidado, división del trabajo y (des) responsabilidades}

La idea de responsabilidad movilizada en la presente reflexión no corresponde a lo que generalmente se entiende como responsabilidad civil, penal o incluso moral cuando se consideran las consecuencias del daño hecho a otro (aun cuando esta dimensión está presente). En base a los resultados de la investigación y haciéndose eco de sus aproximaciones desde la perspectiva del cuidado, remite, por un lado, a la responsabilidad relacional, unida al hecho de que somos sujetos en relación, inscritos, social e individualmente, en redes de cuidado y responsabilidades unos para con otros y, por otro lado, al sentimiento o asunción de responsabilidad que induce el cuidado cotidiano de otro. Ambos aspectos son objetos de tensión y relaciones de poder, en la esfera pública y privada.

\section{Una organización social de cuidado desigual y familista}

Todos dependemos de los cuidados, pero no todos llevamos la misma carga y responsabilidad, sea cual sea el nivel social donde miramos: en la organización social del cuidado; en el seno de la población, donde la carga del cuidado y su falta de reconocimiento afectan mayormente a los grupos más desfavorecidos en función de su pertenencia de sexo, clase y "raza"; dentro de las familias donde recae en mayor medida en mujeres y niñas. Asimismo, los estudios sobre las migraciones de cuidado han puesto de manifiesto la dimensión global de la problemática entre países con diferentes recursos (generalmente de Sur a Norte). Le debemos a Hochschild (2001) haber sacado a la luz algunas dinámicas del cuidado en este contexto global, ya sea en términos de cadenas globales de cuidado (Hochschild, 2000; Parreñas, 2001) o sus consecuencias en términos de trasplante global de los afectos (Hochschild, 2008).

Estas diferentes dimensiones están estrechamente relacionadas entre sí. A diferencia de los países que cuentan con un Estado de bienestar desarrollado, los países latinoamericanos, y Argentina no es una excepción, se caracterizan por un 
modelo de atención familista del cuidado, el cual se apoya principalmente en las estructuras familiares y, en menor medida, en lazos y redes comunitarios (Araujo Guimarães; Hirata, 2020). Las familias que pueden solventarlo, delegan algunas de estas tareas al mercado, sea de manera extra-doméstica (servicios educativos, de salud, gerontológicos) o en el hogar (empleadas domésticas, niñeras, cuidadoras domiciliarias) y apelan asimismo a las solidaridades familiares, para asegurar las necesidades de cuidado de la familia.

Para las familias que no pueden externalizar estas tareas, o bien la mujer no se inserta en el mercado laboral (que a menudo le reserva empleos precarios y mal remunerados) y se dedica al cuidado familiar, o recurren a las solidaridades familiares y/o a servicios comunitarios y/o a servicios asistenciales del Estado. En la mayoría de los casos, la provisión de cuidados implica una articulación entre diferentes actores, en proporciones muy variables según el nivel socioeconómico de la unidad doméstica, su ubicación territorial, las elecciones que hace, mientras la centralidad del rol de la familia y particularmente de las mujeres en el cuidado de los niños más pequeños, sigue siendo socialmente valorado (Faur, 2012). El hecho de que el Estado reserve sus intervenciones para con los más dependientes en aquellos casos en los que "fallaron" los demás recursos (por ejemplo, en el caso de cuidado del adultos mayores), destaca el papel que han de jugar las familias y el mercado en la provisión de cuidado de estas poblaciones.

\section{División del trabajo y de los roles, entre ambigüedades y tensiones}

En las situaciones estudiadas, las familias de clase media, generalmente compuestas por profesionales, emplean a una persona para cuidar a un mayor dependiente. En la mayoría de los casos, se trata de una anciana que vive en su propio hogar, pero que ya no puede vivir sola. Sus hijos no pueden asumir el acompañamiento, delegándolo a una cuidadora remunerada que permanecerá con ella. Se trata en este caso de un cuidado remunerado, pero la vida compartida pesa sobre las relaciones entre las partes y sobre los deberes prestados o asumidos.

En este nivel de análisis, las relaciones entre división del trabajo, roles y responsabilidades son más complejas y ambiguas, y están sujetas a tensiones en varios puntos. Pero, ¿̨qué es lo que delegan las familias? No hay una respuesta única a esta pregunta, que depende de cada organización familiar, de las necesidades de cuidado y de la capacidad de los familiares de reconocer y aceptar el deterioro de sus padres, lo cual no es evidente. Si bien las cuidadoras buscan que la persona mayor atendida atestigüe el cuidado recibido, sean cuales sean las dificultades para lograrlo, no alcanza con hablar de delegación del "trabajo sucio". ${ }^{3}$ En la mayoría de los casos, la familia vive en otro lugar y viene de visita. No hay, estrictamente hablando, división del trabajo concreto del cuidado ni delegación de una u otra de sus tareas, sino de todo lo que resulta necesario para que el asistido viva cotidianamente lo mejor posible. Esto se traduce, para la cuidadora, en estas condiciones particularmente constreñidas, en cierta libertad de acción en la producción del cuidado, así como en cierto limbo (en parte inevitable) en 
torno a las actividades a realizar, fruto de las dimensiones entrelazadas del cuidado, materiales, relacionales y emocionales. Por lo tanto, se trata efectivamente de la responsabilidad del cuidado del otro y, a través de él, de la responsabilidad del otro.

Sin embargo, la división de los roles no es sencilla. Una cosa es delegar las actividades de cuidado y la responsabilidad de un mal cuidado y abuso potenciales (en situaciones en las cuales las condiciones laborales ponen a dura prueba la capacidad de resistencia y de solicitud de quien cuida), otra es reconocer el lugar del afecto y del conocimiento íntimo del otro que se crean en base a ese trabajo. Los relatos revelan situaciones en las que chocan el rol que los familiares desean conservar (con una persona que conocieron cuando era autónoma) y el que conlleva el saber privilegiado que las cuidadoras han desarrollado del otro (de quien anticipan el creciente deterioro). Como, por ejemplo, la esposa que se siente marginada por el papel que su hija le da a una cuidadora para con su esposo, de quien siempre se ocupó. O aquellos hijos que privan a la cuidadora de asistir al entierro de su madre a quien ella cuidó durante años. O esta cuidadora que, su día de descanso, ante la indiferencia que manifiesta la hija de la anciana cuidada, lleva a su asistida a su propia casa, con su familia.

Desde un enfoque de responsabilidad relacional, algunos actores, aquí encarnados por los hijos de clase media y media alta profesional, tienen la posibilidad de delegar la responsabilidad diaria del cuidado (que les correspondería por su lugar en la familia y el modelo familista de cuidados imperante) a mujeres de sectores populares o migrantes. Esta delegación de la responsabilidad cotidiana, así como cierta ignorancia hacia el trabajo de cuidado, son posibles (y se legitiman) por el lugar social que ocupan los familiares y por la contraparte monetaria ("para eso se le paga"), aumentando así la responsabilidad de la cuidadora, que carece de los medios para "comprar un alivio de su carga". Pero, al mismo tiempo, no hay correspondencia entre actividades delegadas, responsabilidad delegada y roles atribuidos por las partes. En otras palabras, existen varias interpretaciones sobre la responsabilidad delegada y asumida, y los roles asociados, íntimamente vinculados a la complejidad del cuidado, su naturaleza por definición "en exceso", así como a las temporalidades de la dependencia atendida.

En general, los hijos se encuentran constreñidos por sus obligaciones laborales y familiares, y visitan a sus padres una o dos veces por semana. Llaman más regularmente y esperan, por cierto, encontrar a su madre o su madre presentable, sin tener que saber demasiado acerca de las "miserias" del trabajo que implica (lo cual tiende a tapar el progreso de la enfermedad). Mantienen una responsabilidad moral como hijos; cuidan a sus padres delegando su cuidado cotidiano a una persona que podrá atenderlo adecuadamente (de lo contrario, actuarán), manteniendo cierta distancia con los aspectos menos gratos del cuidado.

Las cuidadoras, confrontadas con las dificultades del cuidado cotidiano, construyen con su trabajo una relación de responsabilidad como "estar con", 
dirigida hacia el otro, que responde a su propia concepción del cuidado ( ‘ [cuidar] es brindar lo mejor que uno tiene para que la otra persona se sienta tranquila", "El atender a una persona es atenderlo en todo lo que necesite"). Este "estar con", que reivindican, tiende a construirse también en oposición a sus empleadores que delegan une responsabilidad del cuidado que, según ellas, les incumbe, mientras se profundiza la dependencia física, mental, pero también psicológica y emocional del asistido con la cuidadora. Se generan las tensiones mencionadas anteriormente entre los roles, los afectos y la responsabilidad de unos y otros, mientras que, en última instancia, los hijos tienen el poder de "poner" a la cuidadora "en su lugar". Mientras tanto, se mantiene latente la amenaza del accidente que dejaría lugar a la responsabilidad de la cuidadora en su sentido más estricto, como obligación de quien tiene que responder por sus actos y asumir las consecuencias. Tal eventualidad alimenta el miedo constante de las cuidadoras que saben que no pueden controlarlo todo.

\section{Cuidado, trabajo de la relación y compromiso de sí}

Las problemáticas relacionadas con el cuidado y su responsabilidad se deben en parte a las particularidades del trabajo de cuidado en las condiciones analizadas, a la implicación física y psicológica que supone, a la huella que deja.

Lo vimos arriba y podríamos multiplicar los ejemplos, para las cuidadoras su trabajo no consiste en lavar a una persona (como se lavaría un auto) o en hacerla comer (como si fuera un gesto mecánico). Lavar un cuerpo como un vehículo o alimentarlo mecánicamente traduce, al contrario, la pérdida de la dimensión del otro como sujeto y del sentido del cuidado. Por debajo de la parte visible del iceberg, la actividad en apariencia más inocua implica un intenso trabajo sobre uno mismo, sobre el otro, sobre los allegados y sobre la relación de cuidado, dilemas y elecciones morales, entrenamiento y gimnasia perceptiva, etc. Tal complejidad ha sido analizada detenidamente en otros espacios y no se puede reproducir aquí (Borgeaud-Garciandía, 2017). Sin embargo, cabe subrayar que es precisamente a través de ese análisis que se impuso la idea de coerción por responsabilidad y obligación moral, no solo como consecuencia de la delegación por parte de otros, sino como fruto del trabajo mismo. Porque el cuidado no está determinado, se construye sin fijarse nunca. Los efectos perturbadores de la enfermedad, una discusión con un hijo o hija, un pico de angustia, la dosis de la medicación... una nada puede alterar el frágil equilibrio.

Lo que he llamado el "trabajo de la relación" de cuidado (ibidem) representa una parte importante del trabajo, que se refiere a la construcción, día tras día y cada uno de ellos, de las condiciones necesarias para su desarrollo. Se refiere a un intenso trabajo realizado sobre la relación de cuidado, la expresión de los afectos, el entorno espacial y relacional de la persona asistida, elaborando condiciones de desempeño lo más serenas y previsibles posible, que permiten a la vez dar los cuidados y protegerse de los aspectos perjudiciales de la actividad. Esto requiere desarrollar una percepción y conocimiento finos de la persona 
asistida, de su familia y las relaciones entre ellos, de los efectos de la enfermedad y, también, mucha habilidad. Este trabajo, esencial al comienzo de la relación, continúa, más o menos intensivamente, todo a lo largo del empleo (particularmente en caso de enfermedad neurodegenerativa).

El trabajo de la relación, que introduce al otro como un sujeto de cuidado, permite elaborar afectos que protegen contra la objetivación del otro y los posibles efectos de la exasperación y lasitud. La intimidad, el trabajo del cuerpo, la muerte son objeto de resignificación por parte de la cuidadora. Acompañar al otro hasta su deceso, proteger su intimidad perdida, reconocer la vulnerabilidad humana compartida ("porque - dice Rosalba hablando de un asistido que sufre de incontinencia fecal - todos somos seres humanos, todos hacemos lo mismo"), transformar las resistencias en desafíos a su saber-hacer, son algunas de las lecturas ofrecidas por las cuidadoras, y que contribuyen a construir el significado de su actividad y su presencia. Esta postura es necesaria para llevar a cabo su trabajo y afianzarlas en su rol de cuidadoras. Se impone para poder defenderse de las dimensiones fragilizadoras del cuidado, que se naturalizan o simplemente se aceptan, y su carácter socialmente perturbador es resignificado. Sin esta desdramatización y resignificación, que funcionan como diques protectores, el trabajo probablemente resultaría insuperable.

Todo aquello que contribuye a la penosidad particular de este cuidado representa elementos importantes que integran la asunción propia del cuidado de otro. La convivencia continua y el temor de que algo le suceda al mayor, ante lo cual serían justa o injustamente consideradas responsables, refuerzan este sentimiento, pero no es suficiente. El trabajo de la relación y los afectos, el proceso de resignificación positiva del trabajo del cuerpo y la muerte, el conocimiento íntimo del otro a la vez intelectual, psíquico, corporal y afectiva, el tiempo largo y la soledad que permiten desarrollar un cuidado personal y creativo, integran este proceso. Para decirlo de otra manera, y resumiendo lo que se avanzó anteriormente, la responsabilidad como "estar con" se construye con / por el trabajo de cuidado, tanto como respuesta a la dependencia de otro como defensa ante el riesgo de ceder a la irritación y el maltrato. Se refuerza asimismo con la no responsabilización de la persona asistida, cuando ésta ha perdido toda autonomía y no puede responder por sus actos, incluso si son violentos (Molinier, 2013). Detrás de esta señora mayor apacible y pulcra, y un empleo que parece poco demandante, se desarrolla una actividad muy intensa, compleja y delicada, que a menudo deja a las cuidadoras al borde del agotamiento.

Este compromiso personal, aunque defensivo, puede tener efectos igualmente perjudiciales. La penetración subjetiva por el cuidado y la responsabilidad del otro se manifiesta por la continuidad de la atención (llamadas telefónicas, consejos, visitas) el día de descanso, mientras que cada ausencia de la cuidadora, no siempre bien vivida por la persona mayor, se traduce a su regreso por un incremento del cuidado a brindar. Asimismo, las cuidadoras manifiestan descon- 
fianza hacia su remplazante que altera el equilibrio construido; inclusive, algunas renuncian a sus francos para tratar de controlar mejor todo aquello que puede afectar su trabajo y su empleo.

\section{Relaciones de cuidado y responsabilidad, entre dominación y poder}

El trabajo de cuidado remunerado involucra relaciones de poder asimétricas. Las más evidentes son las relaciones de dependencia entre la persona que asiste y la persona asistida, y la asimetría es tanto más importante cuanto que la dependencia es profunda. Las personas que son muy vulnerables por razones de edad o salud están a merced de quienes las cuidan. Estas relaciones de poder y dependencia no son contradictorias con el ejercicio de formas de dominación ejercidas "para el bien" de la persona asistida (Garrau, 2009), o incluso para poder realizar el trabajo de cuidado. Aquí viene nuevamente el trabajo de la relación, que permite un mejor acceso a la persuasión. Esta dominación, obviamente, puede ser excesiva y caer en abusos y malos tratos, un riesgo que aumenta con el aislamiento y cuando las condiciones de trabajo impiden su desarrollo correcto. La posición de poder de las cuidadoras es al mismo tiempo "absoluta" y completamente relativa.

Garrau (2009) destaca una triple dominación que atañe a las cuidadoras: una dominación social estructural vinculada a su posición subordinada en la organización social, en función de líneas de género, clase y "raza"; una dominación derivada de esta posición socialmente dominada y que ubica a la cuidadora en una situación de dependencia económica hacia terceros (su pareja, políticas asistenciales); una dominación psicológica ejercida por la persona asistida (o su familia) que se deriva de la responsabilidad moral asociada con las actividades de cuidado y la necesaria apertura de la cuidadora hacia las necesidades de la persona asistida. Este aspecto sobresaliente de la penosidad de este trabajo puede ir acompañada, según la autora que retoma a Kittay, por una "tiranía de la carga" vinculada a formas de explotación del compromiso de la cuidadora, que no puede ponerle fácilmente fin a la relación. Un estudio micro sociológico de las relaciones de cuidado y dominación completa y complejiza este enfoque. Por ejemplo, cuando estas relaciones entremezclan relaciones de dependencia cruzadas, relaciones de empleo, relaciones de trabajo, relaciones afectivas, todas ellas generadoras de expectativas, esperanzas, reconocimiento o decepción, y que se toman apoyo en las especificidades del trabajo proporcionado (Borgeaud-Garciandía, 2017).

En los casos estudiados, aunque se trata de un trabajo remunerado realizado por una mujer empleada para este fin, las particularidades del empleo la acercan a un "cuidado familiar" cuando éste se produce dentro de un hogar compartido. La cuidadora se encuentra a la vez "adentro" y "afuera", alternativamente íntima allegada y empleada. Se espera de ella el cuidado técnico, material, acompañado por el afecto y la ternura que se supone necesita la persona 
asistida. Sin embargo, rara vez se la invita a ser parte de la toma de decisiones de mayor impacto para esta última (como su institucionalización) y será en tanto empleada que ha fallido que será aprehendida si llegara a sucederle algo mientras se encuentra bajo su responsabilidad (aquí, en el sentido de obligación). Esta situación es fuente de ambigüedades y tensiones entre las cuidadoras y sus empleadores, los hijos de las personas asistidas, subyace en la dinámica de las relaciones de poder e impacta en las tensiones en torno a las responsabilidades adjudicadas y/o asumidas. Desde el punto de vista de la responsabilidad, vuelve la situación aún más compleja, inseparable de las características y efectos del cuidado.

La asunción de la responsabilidad moral por parte de las cuidadoras tiene varias aristas. Sus relatos la justifican apoyándose en dos temas principales: el incumplimiento por parte de los hijos de sus deberes filiales, juzgados con severidad en base a una moral familiar tradicional, ${ }^{4} \mathrm{y}$ el "amor", el cual se refiere menos a un sentimiento que a la obligación moral que conlleva la dependencia, ${ }^{5}$ aligerándola en parte de su peso. Detrás del veredicto sobre los hijos que "abandonan" a sus viejos padres (el abandono como antítesis de la responsabilidad y el amor), o que rechazan o niegan su deterioro, subyacen críticas a las condiciones de trabajo y de empleo, a la vez que las cuidadoras se posicionan como quienes conocen en profundidad el cuidado y el sujeto del cuidado. Los reproches relacionados con las condiciones de empleo, percibidos como poco "nobles" en comparación con los afectos esperados (" ¿Qué puede prevalecer más? - pregunta Estrella - ¿Amor o dinero?), se cristalizan en problemáticas morales, donde pueden ponerse en valor. El conocimiento íntimo del otro integra las relaciones de poder y articula reconocimiento de sí como trabajador y control del empleo. Pues si la dependencia se canaliza de una manera considerada satisfactoria por una relación de cuidado "que funciona", será mucho más costoso (para la persona asistida y para su familia) reemplazar a la cuidadora. Ello, aunque al final, las cuidadoras, constreñidas por las obligaciones morales propias del cuidado dispensado, no pueden "abandonar" la persona asistida, pero si pueden ser despedidas sin previo aviso.

\section{Conclusión}

El deseo de profundizar la reflexión sobre la responsabilidad y el cuidado no es de origen teórico, sino empírico. Nace de lo que uno podría concebir como un núcleo duro del propio trabajo de cuidado, que será tanto más importante cuanto que la dependencia es profunda y la cuidadora aislada. Y contribuye sin duda a colmar la brecha entre responsabilidad como ética del cuidado y responsabilidad como parte del trabajo concreto de cuidado, que aparecen en las experiencias ordinarias de cuidado como las dos caras de una misma moneda.

En los casos analizados, la convivencia, el encierro y la soledad en un contexto de trabajo de alta dependencia tienden a exacerbar las particularidades de lo que hace al cuidado de otro, que encontramos en formas más atenuadas en 
otros profesionales. Así, no se presentarán de idéntica manera según se trabaje a domicilio con un solo asistido, con tres o cuatro personas mayores semi-dependientes unas horas por semana o en una institución geriátrica de larga estadía, en el seno de equipos profesionales que se hacen cargo de varios adultos mayores. Aun así, y a pesar de las exhortaciones a "mantener distancia", el cuidado del otro implica un compromiso de sí, de apertura al otro, sin lo cual el trabajo resultaría insuperable. No se trata de ningún sentimiento positivo innato, sino del resultado del trabajo de cuidado en sí y del trabajo sobre sí mismo que implica la confrontación con la vulnerabilidad humana. El compromiso personal es inevitable, vital y defensivo. Fuente de autovaloración, también puede volverse extenuante e inclusive, en demasía, autodestructivo. Estos diferentes aspectos del trabajo de cuidado se hacen más visibles en las situaciones estudiadas, llevadas al extremo.

Pensar en la responsabilidad, en el corazón del trabajo de cuidado, se refiere a un entrelazado complejo de responsabilidades, que interviene en varios niveles, de acuerdo con sus diversos significados. La responsabilidad relacional y las relaciones de poder ofrecen elementos de respuesta a la famosa pregunta “QQuién se ocupa de qué...?” (Molinier et al., 2009, p.103) que revela la distribución desigual del trabajo de cuidado y politiza la cuestión de su organización, en diferentes escalas. Esta pregunta ha sido ampliamente retomada por los sociólogos. Por otro lado, la pregunta siguiente -" ¿... y cómo? “- (ibidem), partiendo de los puntos de vista de los trabajadores directamente involucrados, es más incómoda y ha sido menos analizada. Resulta más incómoda en tanto plantea preguntas que molestan (relacionadas con el afecto, la moral, la sexualidad, etc. tantas dimensiones embarazosas para las ciencias sociales) e implica, como lo observó Paperman (2013), cuestionar los conocimientos adquiridos y el lugar del "experto sociólogo". Esta pregunta, que toca lo más profundo del trabajo de cuidado, moviliza otra dimensión de la responsabilidad que surge de este trabajo vivo sobre lo humano, preservándolo de la amenaza de deshumanización. Ese "¡y cómo? “, que reenvía a la intimidad del trabajo de cuidado y las resistencias a las que da lugar, también se encuentra íntimamente ligado a la distribución de poder, del trabajo y de las responsabilidades. En este sentido, es a partir de las especificidades, constreñimientos y exigencias del trabajo, más que en términos de servilismo (Memmi, 2016), que podemos entender mejor los compromisos, resistencias y repliegues de las trabajadoras, así como abrirse a la complejidad de las formas de explotación a las que dan lugar, y articulan desigual división social y familiar del trabajo, delegación del cuidado hacia actores socialmente subordinados, y el compromiso de sí y responsabilidad hacia el otro que implica su asunción. Piedra discreta pero angular de la dominación, el compromiso de sí que moviliza el trabajo de cuidado reenvía a la ética del cuidado (o de sí en el cuidado), es, a su vez, fuente de autovaloración e identidad. 


\section{Notas}

1 Sobre los mecanismos de incitación al registro del empleo doméstico y, luego, la implementación en 2013 de la ley 26.844 dicha de Régimen Especial de Contrato de Trabajo para el Personal de Casas Particulares, ver Pereyra (2012) y Esquivel y Pereyra (2017).

2 La primera década del 2000 fue marcada por la campaña de regularización migratoria Patria Grande (2006), que tenía como objetivo regularizar la situación migratoria de las personas miembros del Mercosur, y por la entrada en vigencia de la nueva Ley de Migraciones (Ley 26.871, aprobada en 2004, reglamentada en 2010) que reconoce el derecho de las personas a migrar y la libre circulación de extranjeros miembros del Mercosur.

3 Tal como se vera más adelante, no entienden su actividad como repugnante o degradante. El cuidado realizado y su valor intrínseco llevan a las cuidadoras a construir un significado positivo de lo que se entiende por "trabajo sucio", mientras que vinculan los aspectos menos "nobles" con otros aspectos de su empleo.

4 La familia, idealmente presentada como espacio de amor, cuidado y solidaridad, no se corresponde necesariamente con las experiencias familiares de las cuidadoras, sino que sirve para sostener su posición crítica.

5 Por ejemplo, Estrella, muy poco remunerada por sus empleadores, cuando dice: "Pero fue más que nada, el amor que le llegué a tener a la abuela; fue lo que me hizo quedarme [...]. Porque yo decía "Si yo me voy, ella se enferma" [...] Yo tenía posibilidades de irme a trabajar a otro sitio. (ver el caso de Estrella en Borgeaud-Garciandía, 2017, cap. IV). Sobre el “amor”, cf. Molinier (2005), Borgeaud-Garciandía (2017).

\section{Referencias}

ARAUjO GUIMARÃES, N.; HIRATA, H. (Coord.) El cuidado en América Latina. Mirando a los casos de Argentina, Brasil, Chile, Colombia y Uruguay (título provisório). Buenos Aires: ed. Fundación Medifé, 2020.

BIROLI, F. Responsabilidades, cuidado e democracia. Revista Brasileira de Ciência Politica, n.18, 2015.

BORGEAUD-GARCIANDÍA, N. En la intimidad del cuidado de ancianos dependientes: la experiencia de cuidadoras sin retiro en la Ciudad Autónoma de Buenos Aires. In: PAUTASSI, L.; ZIBECCHI, C. (Org.) Las fronteras del cuidado. Agenda, derechos e infraestructura. Buenos Aires: Biblos, 2013.

Intimidad, sexualidad, demencias. Estrategias afectivas y apropiación del trabajo de cuidado en contextos desestabilizantes. Papeles del CEIC, v.1, papel 148, 2016.

Puertas adentro. Trabajo de cuidado domiciliario a adultos mayores y migración en la Ciudad de Buenos Aires. Buenos Aires: Teseo, 2017. Accesible en: <https:// www.teseopress.com/puertasadentro>.

ESQUIVEL, V.; PEREYRA F. Las condiciones laborales de las y los trabajadores del cuidado en Argentina. Reflexiones en base al análisis de tres ocupaciones seleccionadas. Trabajo y Sociedad, n.28, 2017.

FAUR, E. El cuidado infantil desde las perspectivas de las mujeres-madres. Un estudio en dos barrios populares del Área Metropolitana de Buenos Aires. In: ESQUIVEL, V.; FAUR, E.; JELIN, E. Las lógicas del cuidado infantil. Buenos Aires: IDES, 2012 
GARRAU, M. Le care entre dépendance et domination: l'intérêt de la théorie néo républicaine pour penser une "caring society". Les Ateliers de l'Éthique, v.4, n.2, 2009.

GILLIGAN, C. In a different voice: psychological theory and women development. Cambridge: Harvard University Press, 1982.

GROS, F. Le soin au cœur de l'éthique et l'éthique du soin. Recherche en Soins infirmiers, n.89, 2007.

HOCHSCHILD, A. R. Global Care Chains and Emotional Surplus Value. In: GIDDENS, A; HUTTON, H. (Eds.). On the Edge: Globalization and the New Millennium (p.130-46). Sage Publishers, 2000..

Amor y Oro. In HOCHSCHILD, A. R. La mercantilización de la vida intima. Apuntes de la casa y el trabajo. Madrid; Buenos Aires: Katz Editores, 2008.

MEMMI, D. Aides à domicile et domination rapprochée. La vie des idées, 4 mai 2016.

MOLINIER, P.; LAUGIER, S.; PAPERMAN P. (Ed.) Qu'est-ce que le care? Souci des autres, sensibilité, responsabilité. Paris: Payot \& Rivages, 2009.

MOLINIER, P. Le care à l'épreuve du travail. Vulnérabilités croisées et savoir-faire discrets. In: PAPERMAN, P.; LAUGIER, S. (Dir.) Le souci des autres. Éthique et politique du care. Paris: Raisons Pratiques, EHESS, 2005.

. Le travail du care. Paris: La Dispute, 2013.

MORICE, A. Recherches sur le paternalisme et le clientélisme contemporains: méthodes et interprétations. Mémoire pour l'Habilitation à Diriger des Recherches. Paris: EHESS, 2000.

PAPERMAN, P. Care et sentiments. Paris: PUF, 2013.

PARREÑAS, R. S. Migrant Filipina Domestic Workers and the International Division of Reproductive Labor. Gender and Society, v.14, n.4, 2001.

PEREYRA, F. La regulación laboral de las trabajadoras domésticas en Argentina: situación actual y perspectivas. In: ESQUIVEL, V.; FAUR, E.; JELIN, E. Las lógicas del cuidado infantil. Buenos Aires: IDES, 2012.

PULCINI, E. Donner le care. Revue du MAUSS, v.39, n.1, 2012.

TRONTO, J. Moral boundaries: A Political Argument for an Ethic of Care. London: Routledge, 1993.

. Particularisme et responsabilité relationnelle en morale: une autre approche de

l'éthique globale. In: MOLINIER, P.; PAPERMAN, P. (Ed.) Contre l'indifférence des privilégiés. À quoi sert le care. Paris: Payot \& Rivages, 2013.

RESUMEN - El articulo ofrece una lectura interpretativa sobre la responsabilidad en el trabajo de cuidado a partir del cuidado realizado por mujeres migrantes que trabajan sin retiro con personas mayores muy dependientes en la capital argentina. En particular, se centra en la responsabilidad como asunción de la obligación moral para con el otro vulnerable. Tras dar información sobre el terreno de estudio y la perspectiva de índole más teórica que se pretender enriquecer, se analizan sucesivamente las relaciones entre responsabilidad y división del trabajo, entre responsabilidad y trabajo de cuidado y, fi- 
nalmente, entre cuidado, responsabilidad, poder y dominación. Se destaca el papel que cumple la responsabilidad como parte intrínseca del trabajo de cuidado, y en la complejidad de las tramas de explotación de quienes se dedican a este trabajo, en el marco de relaciones desiguales de poder.

PALABRAS-CLAVE: Responsabilidad, Cuidado, Trabajo de cuidado, Adultos mayores, Ética.

ABSTRACT - The article offers an interpretative reading of the responsibilities associated with care work, based on experiences of live-in caregivers for the elderly in the Argentine capital. In particular, it focuses on responsibility as an assumption of a moral obligation towards the vulnerable. After providing information on this field of study and our preferred theoretical perspective, the relationships between responsibility and division of labor, between responsibility and care work and, finally, between care, responsibility, power and domination are analyzed successively. The article highlights the role of responsibility as an intrinsic part of care work, within the complexity of exploitation patterns that affect those engaged in it and in the context of unequal power relations.

KEYWORDS: Responsibility, Care, Care work, Elderly, Ethics.

Natacha Borgeaud-Garciandia es doctora en sociología de la Universidad Paris 1-Panthéon Sorbonne e investigadora del IICSAL-Flacso/Conicet en Argentina.

@ - natachbg@gmail.com / http://orcid.org/0000-0001-5532-8562

Recebido em 10.12.2019 e aceito em 6.2.2020.

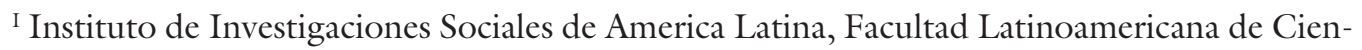
cias Sociales, Buenos Aires, Argentina. 
